\title{
The Studying and Simulating Analysis of the Power Harmonic Measuring Method Based on the Blind Source Analysis
}

\author{
Guo Song-Lin ${ }^{1}$, Wang Jing-Yu ${ }^{1}$ and Liu Fu-Kai ${ }^{1}$ \\ College of Electric and Information Engineering, Heilongjiang Institute of Science \\ and Technology, Harbin, 150022, China \\ gsl63@163.com
}

\begin{abstract}
According to electric power harmonic measuring method of the digital sampling, the simulating was carried out in this paper, thus a kind of based on Newton iterative blind source analysis of Fast - three times the ICA algorithm was Put forward, and its convergence was proved, the simulation analysis of the real-time error and measurement algorithm was finished, its instantaneity and measuring of the algorithm was proved.
\end{abstract}

Keywords: Method of Measurement, Blind Source Analysis, Independent Variable

\section{Introduction}

With the developing of power electronic technology, the using of a large number of nonlinear device on the power supply equipments, a serious impact on power quality of power supply system was caused [1-2]. Along with the developing of computer technology, therefore, the digital sampling technology of domestic and foreign was used in researching of the harmonic detection algorithm. In this paper, the simulation research of harmonic measuring method based on digital sampling technology 'Nuttall window interpolation FFT harmonic analysis method', 'at the same time diagonalization second-order blind identification of harmonic test', 'power grid harmonic detection method based on Mallat wavelet'[3-5]was carried out, the corresponding conclusions was drawn. So the Fast-ICA algorithm for blind source analysis using the Newton iteration method was improved, met the third-order convergence, the iteration times was decreased and the convergence speed was improved, the convergence of the algorithm was proved. The real-time measuring and accuracy of the power harmonic detection was checked by simulation based on Improved Fast-ICA algorithm [6].

\section{The Analysis of Power Harmonic Detection Algorithm}

\subsection{Nuttall Window Interpolation FFT Harmonic Analysis Method}

Set up the expression as input signal

$$
x(t)=\sum_{m=1}^{7} A_{m} \cos 2\left(\pi m f_{1} t+\varphi_{m}\right)
$$

In the formula: The fundamental frequency $f_{1}=50 \mathrm{~Hz}, A_{m}, \varphi_{m}$ are the amplitude and initial phase of fundamental wave and all other harmonic. Set the sampling frequency was $10 \mathrm{KHZ}$, the sampling points was 2048 points (about 10 fundamental wave cycle).

The diagram for simulation process and simulation error curve of Nuttall window with interpolation FFT algorithm block was respectively shown in Figure 1 and Figure 2. 


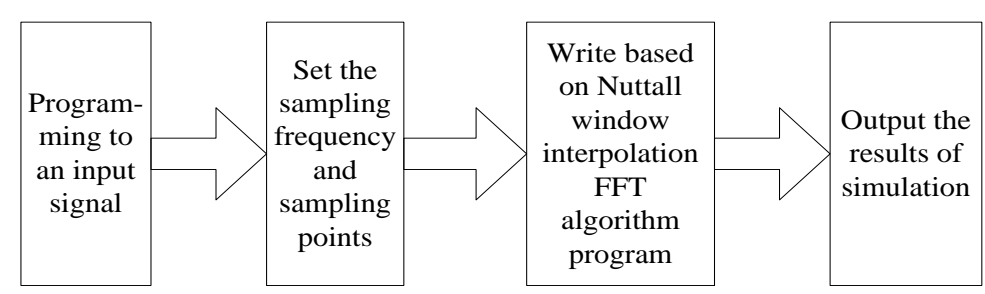

Figure 1. The Simulation Experiment Flow Chart

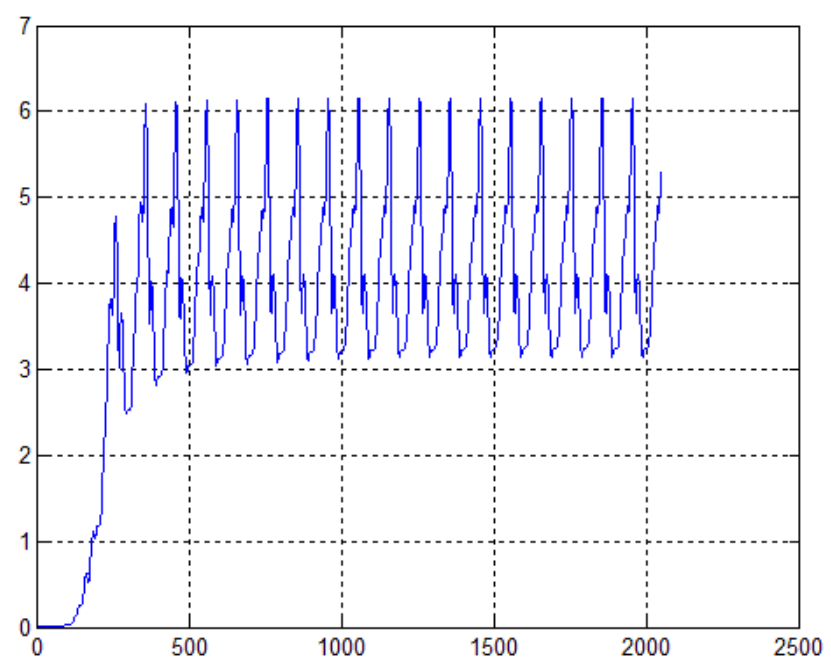

Figure 2. Nuttall Window Interpolation FFT Method Error Curve Graph

To a certain extent, the frequency of leakage was decreased by Nuttall window interpolation FFT harmonic analysis method, the detection precision of the harmonic signal was improved, but the detection error became larger.

\subsection{At the Same Time Diagonalization Second-Order Blind Identification of Harmonic Test}

$\mathrm{X}$ (t) signal contain 3, 5, 7 times harmonic, constitute of $\mathrm{x}(\mathrm{t})$ every harmonic waveform is shown in Figure 3.

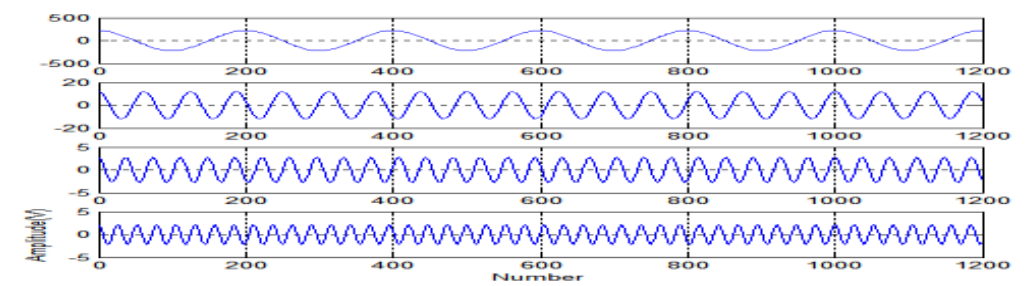

Figure 3. Constitute every Harmonic Waveform Figure of $x(t)$

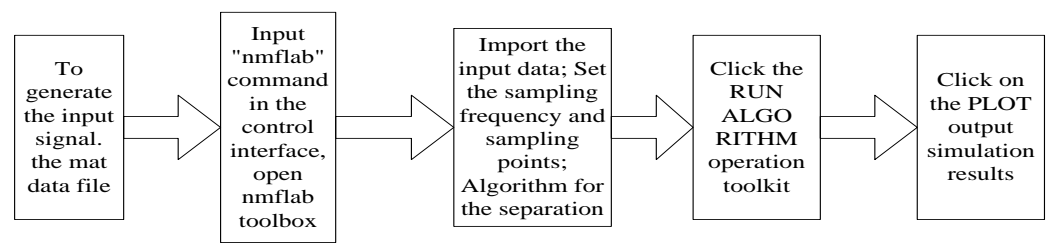

Figure 4. SOBI Algorithm Simulation Flow Chart 


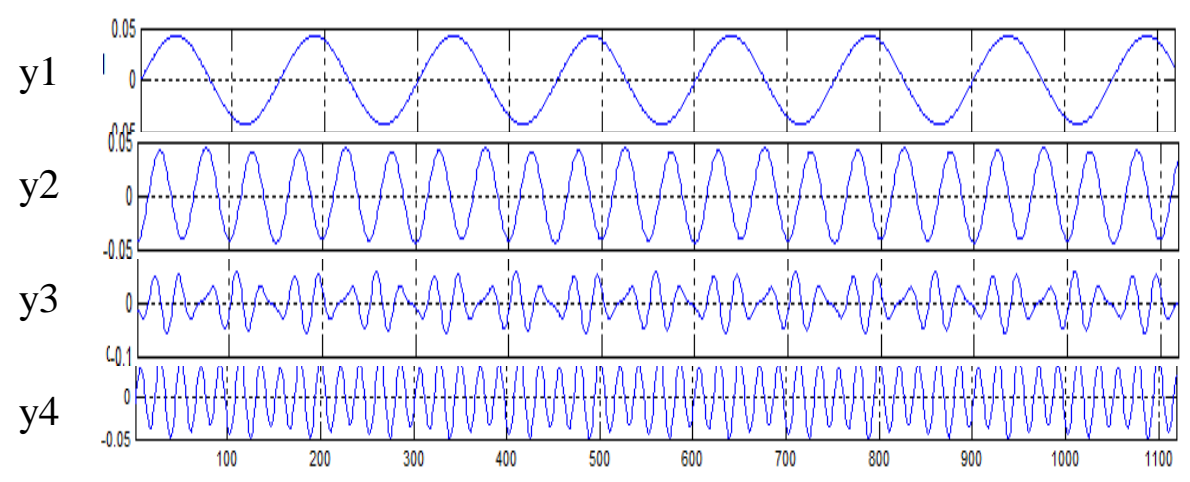

Figure 5. SOBI Separation Results

The frequency of y1-y4:50.0Hz, $150.1 \mathrm{~Hz}, 278.9 \mathrm{~Hz}, 359.2 \mathrm{~Hz}$ was calculated. The average error is about 3.5\%. And, the harmonic signals could be isolated by the SOBI algorithm through the Figuring 5. However, its errors of measuring was bigger.

\subsection{Power Grid Harmonic Detection Method based on Mallat Wavelet}

Set the input signal to a non steady state harmonic signal for $\mathrm{x}(\mathrm{t})$, as shown in Figure 6, the expression of time domain waveform mathematical is

$$
x(t)= \begin{cases}\sin (100 \pi t) & 0 \leq t \leq 0.02 \\ \sin (700 \pi t) & 0.02 \leq t \leq 0.1\end{cases}
$$

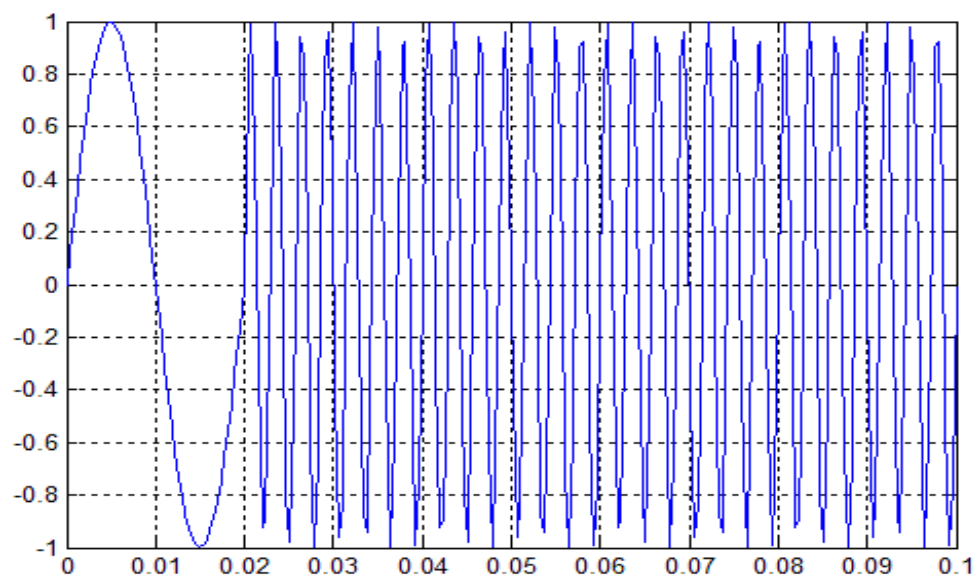

Figure 6. Non-stationary Signal Time Domain Graph

The flow chart of wavelet detecting programs on decomposition and reconstruction of $x(t)$ in Figure 7.

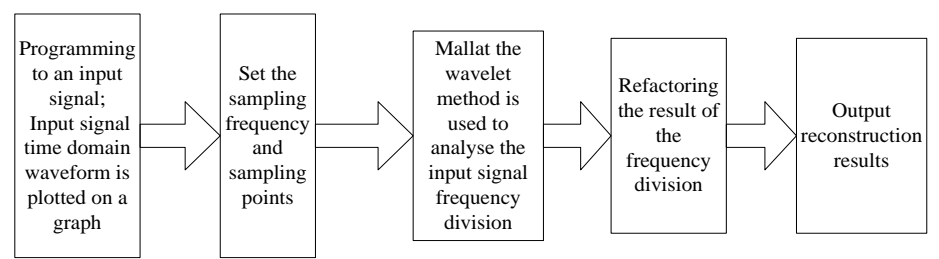

Figure 7. Flow Chart of the Simulation 
The low frequency and high frequency coefficient of $\mathrm{X}(\mathrm{t})$ through the processing of Mallat wavelet algorithm was shown in Figure 8.

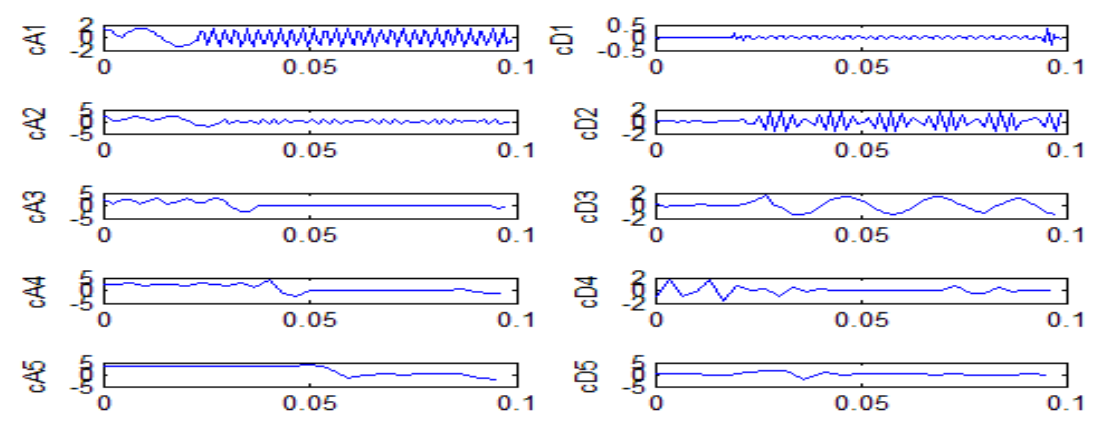

Figure 8. Processed Low, High Frequency Coefficient

The location of the discontinuities was easy to find from the figure 8 in high frequency section (i.e., the details of the waveform) after the processing of Mallat wavelet transform algorithm. A discontinuity point in $0.02 \mathrm{~s}$ was seen clearly within the high frequency coefficients $\mathrm{cD} 1$, and produces a shock signal.

The basis wave and harmonic signal could be reconstructed through wavelet decomposition coefficient. Original signal contains only 7 times harmonic signals, and the frequency signal of $350 \mathrm{~Hz}$ was contained in the frequency band of high frequency coefficients cD3. Therefore, 7 times harmonic signal could be reconstructed based on cD3 through type (2). In a similar way, the basis wave was contained in the frequency band of cA4, which could reconstructed the signal. The results of reconstruction was shown in Figure 9, in Figure 10.
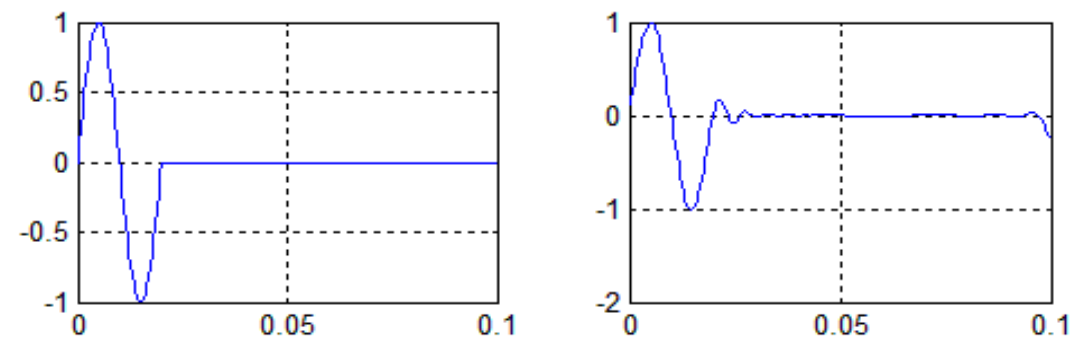
a) Original Fundamental Signal
b) Reconstruction of Fundamental Signal

Figure 9. The Original Base Wave Shape and Reconstruction of Fundamental Wave Signal
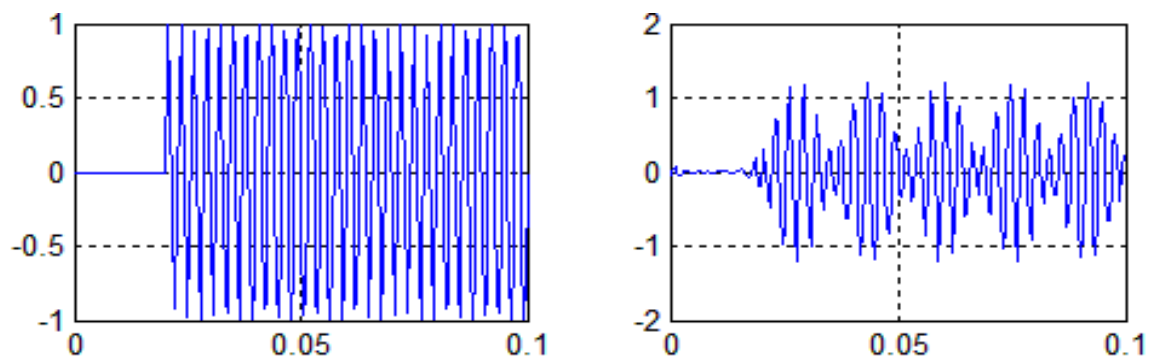

a) Original 7 times harmonic signal

b) Reconstruction of 7times harmonic signal

Figure 10. The Original 7 Times Harmonic Wave Shape and Reconstruction of 7 times Harmonic Wave Signal 
Through the analysis of simulating, harmonic signal could be completely separated through the harmonic detection algorithm based on Mallat wavelet, but the time of testing was longer.

So, the harmonic analysis method based on FFT simpling principle was a convenient calculation. But the accuracy and real-time of the harmonic detection was in general; The amplitude and the output sequence of uncertainty and a certain error were appeared by using at the same time diagonalization second-order blind identification of harmonic test; Harmonic signal could be completely separated through the harmonic detection algorithm based on Mallat wavelet, but the time of testing was longer, unable to meet the real-time requirements.

\section{Independent Component Analysis}

ICA is used only by the output data $\mathrm{X}$ of the multichannel system to determine the input $\mathrm{S}$ and system's transfer function H[7-8]. Mathematical model of ICA in the form of a matrix can be expressed as

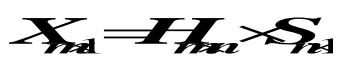

In the formula, Column vector $\mathrm{S}(\mathrm{t})$ is unknown source signals; $\mathrm{H}$ is $\mathrm{m} * \mathrm{n}$ dimensional matrix, usually referred to as a mixed matrix; $\mathrm{X}(\mathrm{t})$ is column vector composed of $\mathrm{m}$ kind of the observation signal. When applications need to solve the separation matrix $\boldsymbol{W}=\left(\boldsymbol{w}_{i j}\right)_{\boldsymbol{m} \times \boldsymbol{n}}$, isolated the source signal. In the absence of interfering signal, through the observation signal $\mathrm{X}(\mathrm{t})$ and separating matrix $\mathrm{W}$ multiplication can be calculated $\mathrm{Y}(\mathrm{t})[9]$, as the estimate of the source signal $\mathrm{S}(\mathrm{t})$, that is

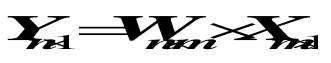

\section{Improved Fast-ICA Algorithm}

Improved algorithm was mainly composed of signal preprocessing and independent component extraction unit, its structure was shown in figure 11, which, for the input variables $\mathrm{X}$ and $\mathrm{Y}$ to have improved Fast-ICA blind source separation algorithm separate

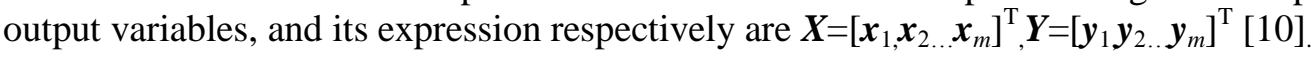

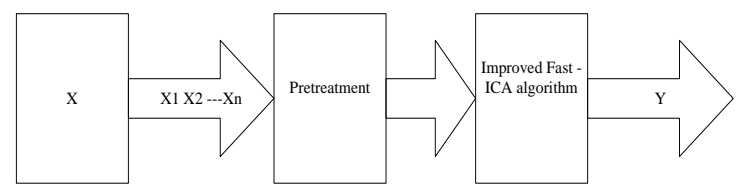

\section{Figure 11. Improved Fast-ICA Algorithm Structure}

\subsection{Input Variable Pretreatment Unit}

Input variable pretreatment unit consists of two parts, spheroidizing and bleaching processing. Spheroidizing is refers to the input variable minus average, making it the number of zeros of the signal; Bleaching is the input variable linear transformation for a unit variance, and unrelated to the components in the signal.

\subsection{The Correlation Criterion of Separation of Variables $Y$}

Algorithm adopts the negative entropy as a criterion of the non-Gaussian nature, provide the separated variable $\mathrm{Y}$ the relevance judgment, to determine the non-Gaussian of $Y$ (independence). Set the component of separated variable $Y$ to $y$, its negative entropy $\mathrm{J}(\mathrm{y})$ is defined as 


$$
\boldsymbol{J}(\boldsymbol{y}) \propto\left[\boldsymbol{E}\{\boldsymbol{G}(\boldsymbol{y})\}-\boldsymbol{E}\left\{\boldsymbol{G}\left(\boldsymbol{y}_{\text {gauss }}\right)\right\}\right]^{2}
$$

In the formula, $\boldsymbol{y}_{\text {gauss }}$ and y hold the same variance of Gaussian variables; $G$ is a random of the quadratic function, the greater of non-Gaussian component of $y$, the smaller the value of $\boldsymbol{E}\left\{\boldsymbol{G}\left(\boldsymbol{y}_{\text {gauss }}\right)\right\}$ is, the greater the negative entropy $\boldsymbol{J}(\boldsymbol{y})$. After the measurement of signal preprocessing to the input $\mathrm{x}$, based on negative entropy criterion, find matrix $\boldsymbol{W}$, made the negative entropy $\mathrm{J}(\mathrm{y})$ biggest [10].In extreme value $\boldsymbol{E}\{\boldsymbol{G}(\boldsymbol{y})\}=\boldsymbol{E}\left\{\boldsymbol{G}\left(\boldsymbol{W}^{\boldsymbol{T}} \boldsymbol{x}\right)\right\}$, makes $\boldsymbol{E}\left\{\boldsymbol{G}\left(\boldsymbol{W}^{\boldsymbol{T}} \boldsymbol{x}\right)\right\}$ biggest. Therefore, the $E\{\mathbf{G}(\mathrm{y})\}$ derivative is

$$
E^{\prime}\left\{G\left(W^{T} x\right)\right\}=E\left\{x \cdot g\left(W^{T} x\right)\right\}=0
$$

In the formula, $g(\cdot)$ as the objective function of the derivative of $G(\cdot)$. Sets the component of input variable $\mathrm{X}$ of $\mathrm{t}$ time to $\mathrm{x}_{\mathrm{t}}$, by Newton's iterative theorem type available $t+1$ times the weight of the input variables $X$

$$
x_{t+1}=x_{t}-\left(y_{t} / y_{t}^{\prime}\right), t=0,1,2, \ldots
$$

In the formula, $y_{t}$ is output after the separation components; $y_{t}^{\prime}$ for its derivatives. As a result, $\mathrm{t}$ time separation matrix of the input variables of the algorithm.

$$
W_{t}=E\left\{x \cdot g\left(W_{t-1}^{T}\right)\right\}-E\left\{g^{\prime}\left(W_{t-1}^{T} x\right)\right\} W_{t-1}
$$

In the formula, $\boldsymbol{W}_{t-1}^{\mathrm{T}}$ for the separation of time $\mathrm{t}-1$ matrix transpose of the matrix. Therefore, the separated matrix is

$$
W=W_{t} /\left\|W_{t}\right\|
$$

\subsection{Improved Fast -ICA Algorithm}

In the original of Fast-ICA algorithm, and adopt the second-order Newton iterative algorithm to deal with data. It could be seen from the type (7), when $y_{t}=0, y_{t}^{\prime} \neq 0$, when Newton iterative algorithm was used in the data processing [11] of the second order convergence. Due to the superiority of third-order Newton algorithm in convergence speed, so to modify type (7), satisfies the third-order convergence, to improve its convergence speed. Correction form for

$$
\left\{\begin{array}{c}
x_{n+1}^{*}=x_{n} \frac{f\left(x_{n}\right)}{f(x)} \\
x_{n+1}=x_{n} \frac{f\left(x_{n}\right)}{6}\left[\frac{1}{f\left(x_{n}\right)}+\frac{4}{f\left(x_{n}+x_{n+1}^{*}\right) / 2}+\frac{1}{f\left(x_{n+1}^{*}\right)}\right] \\
\mathrm{n}=0,1,2 \ldots 。
\end{array}\right.
$$

By type (7) (9), improved Fast-ICA algorithm of matrix of the input variable separation is

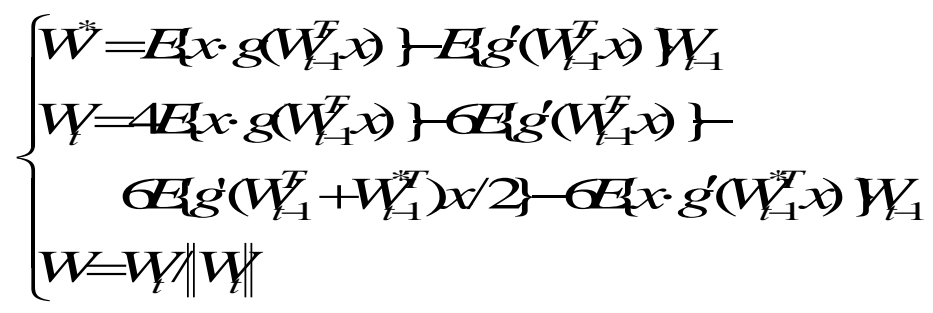




\subsection{Proof of Convergence}

Theorem $\mathrm{f}(\mathrm{x})(I \rightarrow R)$ is the open interval I within a four order differentiable function, if $a \in \boldsymbol{I}$ is the single of $\mathrm{f}(\mathrm{x})=0$, and sufficiently near a, defined by type (10) modifying Newton iterative method for error equation is $\boldsymbol{e}_{n+1}=\frac{1}{3 !} \frac{f^{\prime \prime 2}(a)}{f^{\prime 2}(a)} e_{n}^{3}+\boldsymbol{O}\left(\boldsymbol{e}_{n}^{4}\right)$ [12-13].

Prove : if $\boldsymbol{e}_{\boldsymbol{n}}=\boldsymbol{x}_{\boldsymbol{n}}-\boldsymbol{a}, \quad \boldsymbol{F}(\boldsymbol{x})=\boldsymbol{x}-\frac{\boldsymbol{f}(\boldsymbol{x})}{6}\left[\frac{1}{\boldsymbol{f}^{\prime}(\boldsymbol{x})}+\frac{4}{\boldsymbol{f}^{\prime}((\boldsymbol{x}+\boldsymbol{y}) / 2)}+\frac{1}{\boldsymbol{f}^{\prime}(\boldsymbol{y})}\right]$ Among the , $\boldsymbol{y}=\boldsymbol{x}-\frac{\boldsymbol{f}(\boldsymbol{x})}{\boldsymbol{f}^{\prime}(\boldsymbol{x})}$, Type (10) could be expressed as $\boldsymbol{x}_{n+1}=\boldsymbol{F}\left(\boldsymbol{x}_{\boldsymbol{n}}\right)$, known $\boldsymbol{f}(\boldsymbol{x})$

Continuous differentiable, $\boldsymbol{f}(\boldsymbol{a})=0, \boldsymbol{f}^{\prime}(\boldsymbol{a}) \neq 0 \mathrm{~F}(\mathrm{x})$ at a point in Taylor's formula, $\boldsymbol{F}\left(x_{n}\right)=\boldsymbol{F}(\boldsymbol{a})+\boldsymbol{F}^{\prime}(a) \boldsymbol{e}_{n}+\frac{\boldsymbol{F}^{\prime \prime}(\boldsymbol{a})}{2 !} \boldsymbol{e}_{n}^{2}+\frac{\boldsymbol{F}^{\prime \prime \prime}(\boldsymbol{a})}{3 !} \boldsymbol{e}_{n}^{3}+\boldsymbol{O}\left(\boldsymbol{e}_{n}^{4}\right)$ Among them,

$$
f(a)=0, y(a)=a, y^{\prime}(a)=0, y^{\prime \prime}(a)=\frac{f^{\prime \prime}(a)}{f^{\prime}(a)}
$$

Through calculation can get $\boldsymbol{F}(\boldsymbol{a})=\boldsymbol{a}, \boldsymbol{F}^{\prime}(\boldsymbol{a})=0, \boldsymbol{F}^{\prime \prime}(\boldsymbol{a})=0, \boldsymbol{F}^{\prime \prime}(\boldsymbol{a})=\left(\frac{f^{\prime \prime}(\boldsymbol{a})}{f^{\prime}(\boldsymbol{a})}\right)^{2}$

The iterative formula is

$$
\begin{gathered}
\boldsymbol{x}_{n+1}=\boldsymbol{F}\left(\boldsymbol{x}_{n}\right)=\boldsymbol{a}+\frac{1}{3 !} \frac{\boldsymbol{f}^{\prime 2}(\boldsymbol{a})}{\boldsymbol{f}^{\prime 2}(\boldsymbol{a})} \boldsymbol{e}_{n}^{3}+\boldsymbol{O}\left(\boldsymbol{e}_{n}^{4}\right) \\
\boldsymbol{e}_{n+1}=\frac{1}{3 !} \frac{\boldsymbol{f}^{\prime 2}(\boldsymbol{a})}{\boldsymbol{f}^{\prime 2}(\boldsymbol{a})} \boldsymbol{e}_{n}^{3}+\boldsymbol{O}\left(\boldsymbol{e}_{n}^{4}\right) \\
\text { That is } \lim _{n \rightarrow \infty} \frac{\boldsymbol{e}_{n+1}}{\boldsymbol{e}_{n}^{3}}=\frac{1}{3 !} \frac{\boldsymbol{f}^{\prime 2}(\boldsymbol{a})}{\boldsymbol{f}^{\prime 2}(\boldsymbol{a})}=\boldsymbol{C},
\end{gathered}
$$

So the modified iterative formula is 3 order convergence.

\section{Simulation and Result Analysis}

\subsection{Experiment 1}

The signal of power grid harmonics was seriously influenced by 3,5, 7 times harmonic signal, therefore, the experiment detect contain 3, 5, 7 times harmonic signals to show that the real-time performance and the accuracy of algorithm.

Set sampling points to 920 , sampling frequency of $7500 \mathrm{~Hz}$, the input signal to contain $3,5,7$ times for harmonic expressions is

$$
\begin{gathered}
d(t)=\sin (x 50 t)+\frac{1}{3} \sin (x<15 \theta)+ \\
\frac{1}{5} \sin (\times 250)+\frac{1}{7} \sin (\times 350)
\end{gathered}
$$


The input signal time domain waveform as shown in Figure 12.

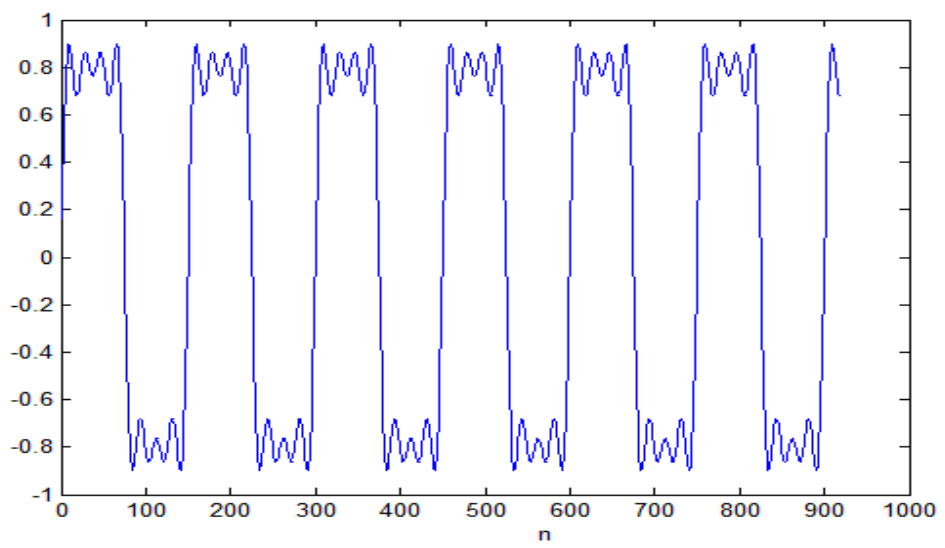

Figure 12. Input Signal

The signal $d(t)$ was respectively measured by using original Fast-ICA algorithm and the improved Fast -ICA algorithm, the experimental results were shown in figure 13, 14. Figure 13 was the result of separation of the input signal to the original algorithm, from top to bottom, the separation results of the basis wave, 3, 7 and 5 harmonic in turn; Figure 14 was the result of separation of the input signal to the improved algorithm, from top to bottom, the separation results of 7, 3,5 harmonic and the basis wave in turn;

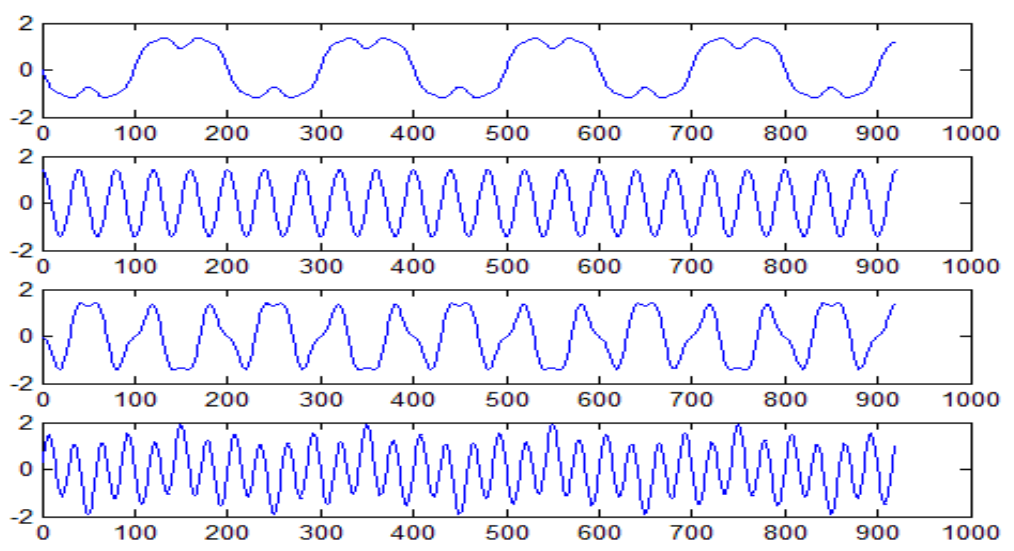

Figure 13. Fast-ICA Separetion Results

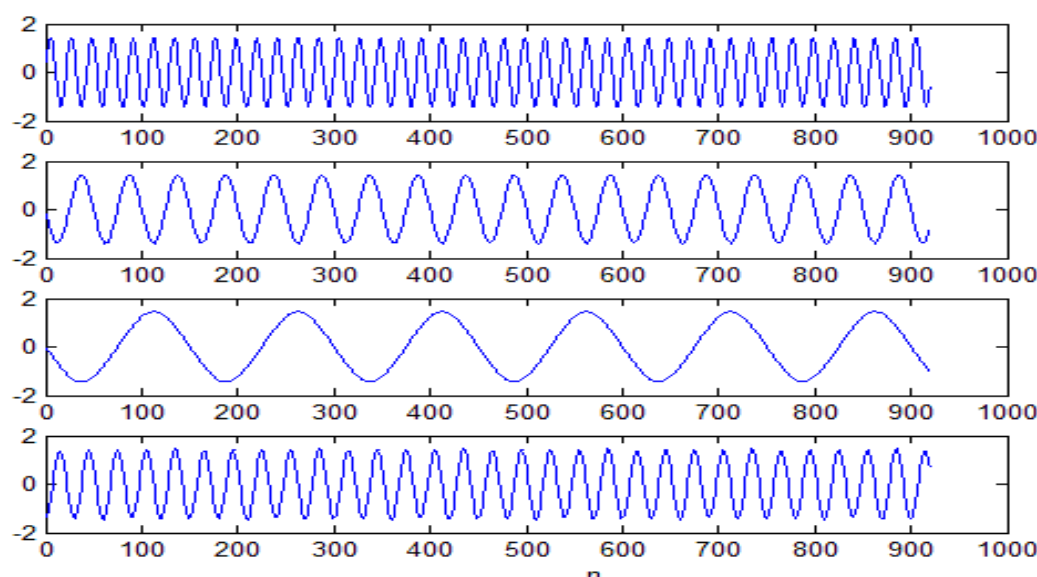

Figure 14. Improved Fast-ICA Separation Results 
To check the accuracy of the improved algorithm, compared with the results of fundamental wave separation between the origin algorithms and the improved algorithms, as shown in Figure 15.

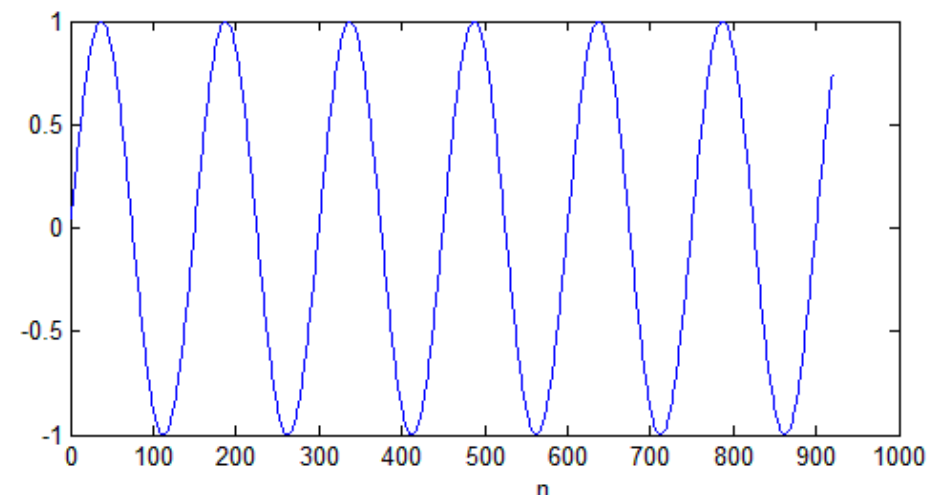

(a) $50 \mathrm{~Hz}$ Fundamental Wave Signal

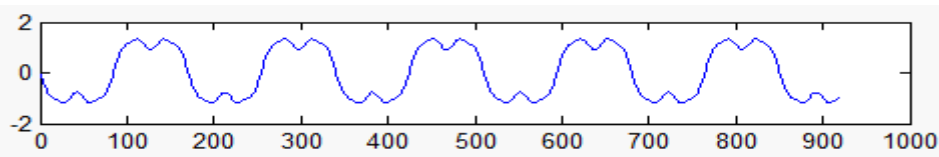

(b) Fast - ICA Separation of Base Wave Signal

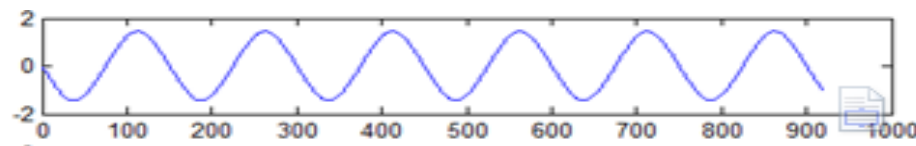

(c) Improved Fast - ICA Separation of Base Wave Signal

Figure 15. Two Methods of the Base Wave Separation Results Contrast

From Figure 15, the contrast of separation results between two methods of the base wave, the separated accuracy of the harmonic signal base on Fast-ICA blind source separation algorithm was improved significantly.

\subsection{Experiment 2}

Utilizing the improved algorithm for harmonic signal with amplitude and phase shift was measured to verify the practicability of the algorithm.

A mixed signal for $\mathrm{x}(\mathrm{t})$, and sampling frequency for $7500 \mathrm{~Hz}$, expression for the signal is

$$
\begin{gathered}
x(t)=22000 \cos 2 \times 50000 t)+ \\
1200 \cos 2 \times 15000 t+20 \pi / 180+ \\
270 \cos 2 \times 25000 t+40 \pi / 180+ \\
2.10 \cos 2 \times 35000 t+60 \pi / 180+ \\
0.30 \cos 2 \times 45000 t+80 \pi / 180
\end{gathered}
$$

Signal waveform is shown in Figure 16. 


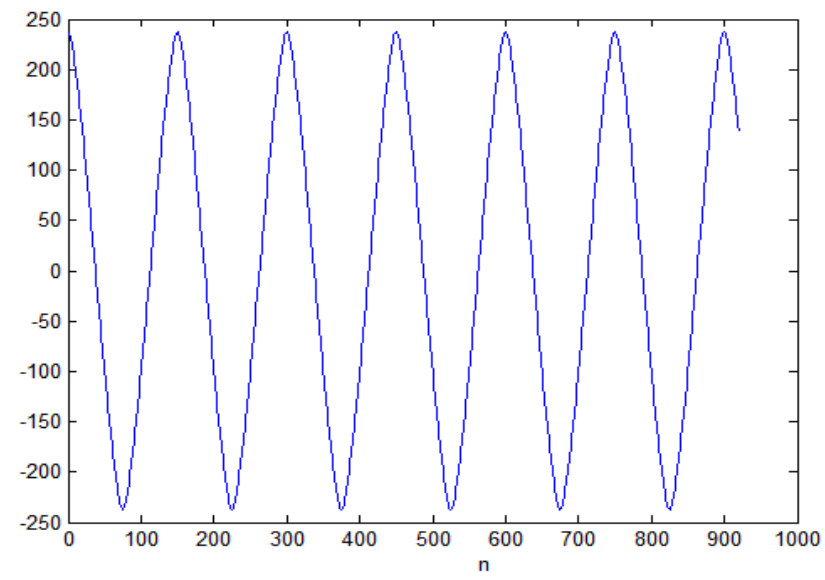

Figure 16. Input Signal

The result is shown in Figures 17, 18 by using the Fast -ICA algorithm and improved Fast - ICA algorithm to deal with input signal.

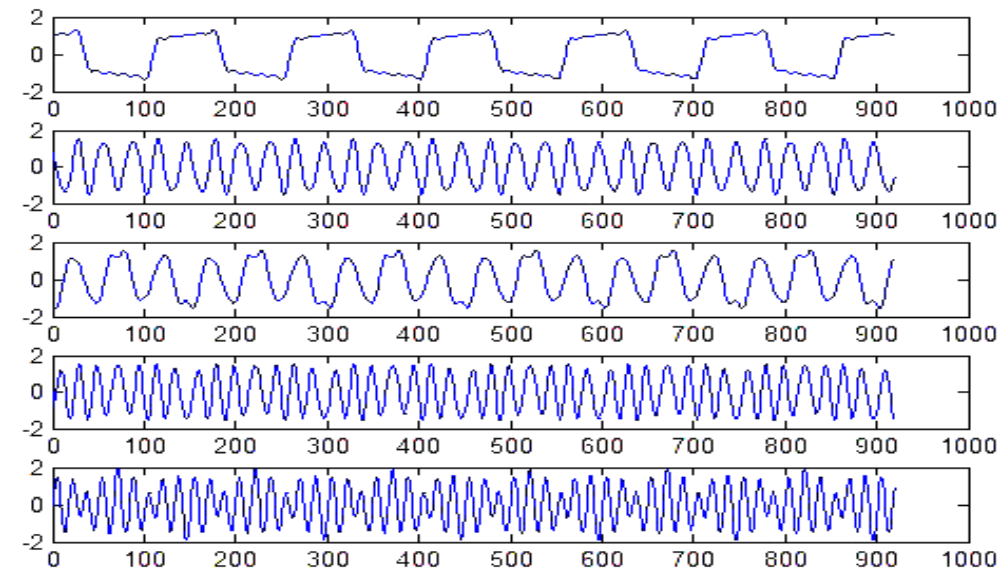

Figure 17. Fast-ICA Separation Results

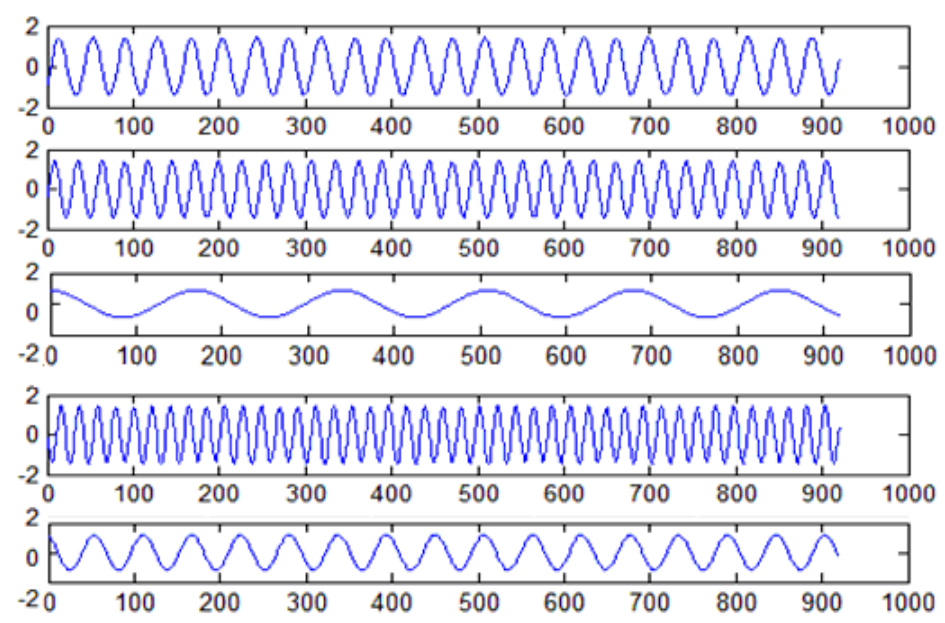

Figure 18. Improved Fast-ICA Separation Results 


\subsection{Improved Algorithm Performance Analysis}

Through the experimental results of Figures 13, 14, 15, 16, showed that the harmonic signal could be extracted from the mixed signal by the improved algorithm, with harmonic detection capabilities. The real-time and accuracy analysis as follow:

(1) In terms of detection real-time, the Newton iterative convergence order was improved, so the data processing speed of algorithm was improved, the processing time was decreased. In the results of experiment 1 , the separating signal operation time through the original algorithm was about $0.8 \mathrm{~s}$, through the improved algorithm was about $0.5 \mathrm{~s}$; In experiment 2 , the separating signal operation time through the original algorithm was about $1.1 \mathrm{~s}$, through the improved algorithm was about $0.7 \mathrm{~s}$. From the test results of Table 1, it could be seen that the speed increased by $37 \%$ on average on the real-time detection through this article optimization algorithm under the same experimental conditions than the original, the detection speed through the ICA algorithm was improved, faster than the original algorithm. Compared the running time between two kinds of algorithm, such as Table 1.

Table 1. Two Experiment Contrast

\begin{tabular}{ccc}
\hline $\begin{array}{c}\text { Algor } \\
\text { ithm }\end{array}$ & Fast-ICA & $\begin{array}{c}\text { Improved } \\
\text { Fast-ICA }\end{array}$ \\
\hline $\begin{array}{l}\text { Experi } \\
\text { ment } 1\end{array}$ & $0.8386 \mathrm{~s}$ & $0.5263 \mathrm{~s}$ \\
\hline $\begin{array}{l}\text { Experi } \\
\text { ment 2 }\end{array}$ & $1.1458 \mathrm{~s}$ & $0.7169 \mathrm{~s}$ \\
\hline
\end{tabular}

(2) In terms of the accuracy of the separation, the basis wave as demonstrated with the improved Fast ICA algorithm is better than the original Fast - ICA algorithm from Figure 15.

The sampling points was 4000 , it could be seen that the error curve contrast diagram between the improved algorithm and the original algorithm from figure 19, and the detection accuracy improved more. In conclusion, the improved algorithm can not only effectively to separate various harmonic signals, and the accuracy and real-time performance was better than the original Fast ICA algorithm. To test the harmonic signal by using the improved method is feasible.

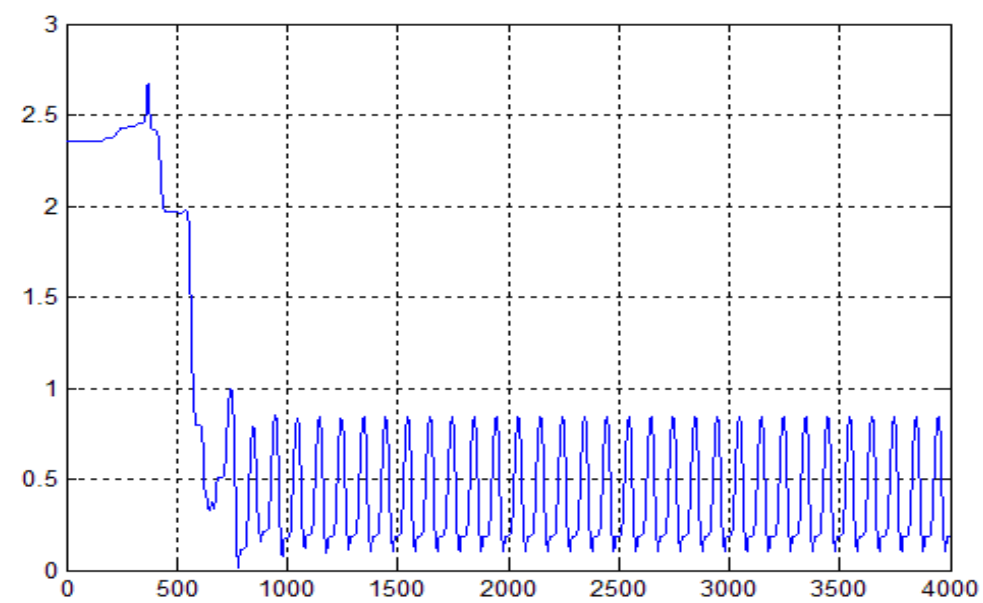

a) Improved Fast-ICA algorithm 


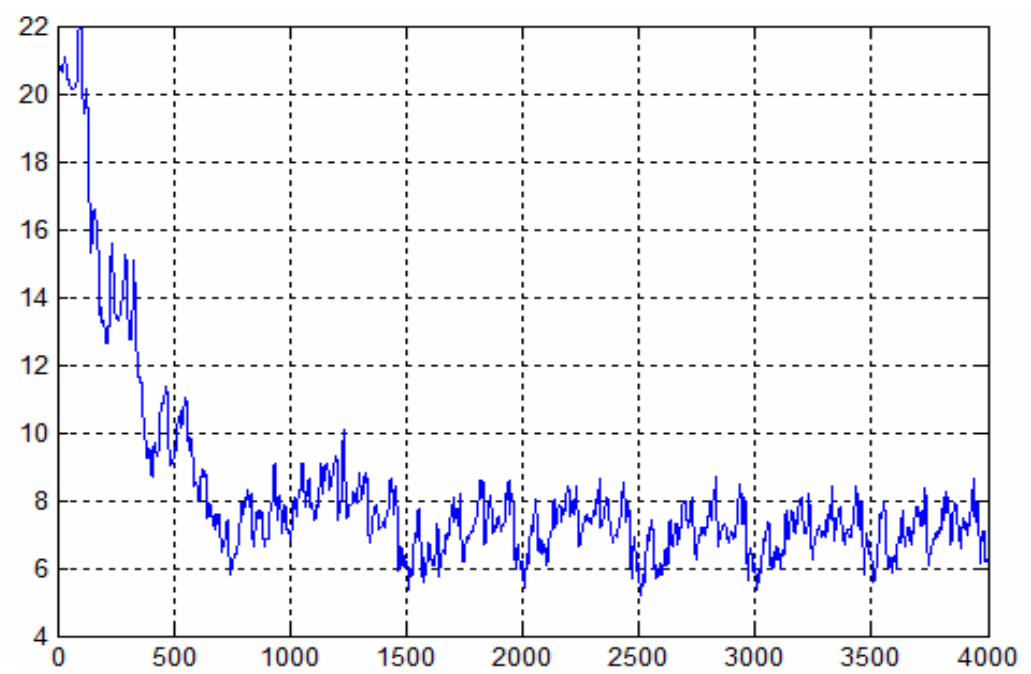

b) Original Fast-ICA Algorithm

\section{Figure 19. Improved Algorithm Error Graph Compares with the Original Algorithm}

\section{Conclusions}

In this paper, the simulation research of harmonic measuring method based on digital sampling technology 'Nuttall window interpolation FFT harmonic analysis method', 'at the same time diagonalization second-order blind identification of harmonic test', 'power grid harmonic detection method based on Mallat wavelet' was carried out, the corresponding conclusions was drawn. Such as: The harmonic analysis method based on FFT was simple, calculated was convenient. Because of its defects, the accuracy and real-time performance of FFT algorithm for harmonic detection was general; By using the diagonalization second-order blind identification algorithm, it could be more effective for harmonic detection, but blind source separation algorithm was lack of a large number of a prior information, so it will appear that the amplitude and the output sequence of uncertainty and a certain error; The harmonic detection algorithm based on Mallat wavelet could complete the harmonic signal separation, but the testing time was long , and unable to meet the real-time requirements.

The Newton's iteration method based on Fast-ICA algorithm was improved, it meets the third-order convergence, the convergence speed was improved. Through the mathematical proof of the algorithm and the computer simulation experiment can draw the following conclusion: The algorithm based on negative entropy rule of great independence realize blind signal separation, the number of iterations was decreased, the convergence speed was improved, and holds the real-time performance. Algorithm could detect the similar grid harmonic signal, and was practical.

\section{References}

[1] X. X. Ning, "Power quality analysis and control". Beijing: China electric power press, (2004).

[2] V. H. "Nonlinear independent component analysis using ensemble learning" Theory International Workshop on Independent Component Analysis and Blind Signal Separation (ICA2000), Helsinki, Finland, (2000).

[3] L. Xiao-Min, "Study on windowed Fourier transform algorithm and its improvement with interpolated method for harmonic detection" J. Journal of Electric power Science and Technology, vol. 22, no.3, (2007), pp. 36-40.

[4] Z. Long-hua, F. Qing and Y. Shi-jie, "Harmonic detection based on wavelet transform", J. Proceedings of the CSU-EPSA, vol. 22 no. 1, (2010), pp. 80-85. 
[5] G. Xu-Han, "Application of wavelet neural network to power harmonic detection", J. Science Technology and Engineering, vol. 10, no. 11, (2010), pp. 2 784-2 788.

[6] W. Kun, "Research on the blind source Separation problems", J. Science \&Technology information, vol. 1 no. 29 (2008), pp. 529-551.

[7] A. Kachenoura, L. Albera and L. Senhadji, "A potential tool for basic system”, J. Signal Processing, vol. 25, no. 1, (2008), pp. 57-68.

[8] C. Gouy-Pailler, M. Congedo and C. Brunner, "Non-stationary Brain Source Separation for Multiclass Motor Imagery", J.IEEE Transactions on Biomedical Engineering, vol. 57, no. 2, (2010), pp. 469-478.

[9] L. T. Duarte, B. Rive and C. Jutten, "Blind extraction of smooth signals based on a second-order frequency identify algorithm,” J .Signal Processing Letters, Signal Processing Letters, IEEE, vol. 17, no. 1, (2010), pp. 79-82.

[10] W. Ji, W. Nian and W. Lian. "Harmonic detection of power quality based on improved Fast-ICA", J. Power System Protection and Control, vol. 38, no. 18, (2010), pp. 126-130.

[11] H. Xu-Chu, S. Yu-cheng and B. Xue-Song, "Introductory tutorial of computational mathematics". Shanghai: people's education press, (1981).

[12] A. H. Rinen, J. Karhune,. "Independent Component Analysis". New York: Wiley, (2001).

[13] B. Xue-Song, "Numerical methods". Beijing: higher education Press, (1990).

[14] Z. Hua, F. Da-zhen and P. Ji-yong. "A time-domain blind convolution separation algorithm for voice signals based on second-order statistics", J. Journal, vol. 32, no. 5, (2010), pp. 1 083-1 087.

[15] Z. Ling and Z. Xian-Da. "An overview of blind channel estimation Algorithms for MIMO-OFDM systems". J. Journal of electronics, vol. 35, no. 6A, (2007), pp. 1-6. 
International Journal of Signal Processing, Image Processing and Pattern Recognition Vol.6, No.6 (2013) 